\title{
The importance of elevated masticatory forces on the stability of maxillary expansion
}

\author{
M. Makaremi ${ }^{1}$, K. Zink ${ }^{2}$, F. de Brondeau ${ }^{3}$ \\ 1 Qualified Specialist in Dental and Facial Orthopedics, Independent Practitioner, \\ Researcher in Anthropology \\ 2 Post-doctoral Fellow in Biological Anthropology, Harvard University \\ 3 Qualified Specialist in Dental and Facial Orthopedics, Independent Practitioner, University Lecturer
}

SUMMARY:

What have our young patients eaten in the past 24 hours? Probably cooked, prepared, or even finely chopped foods, that require limited masticatory action compared to what our craniofacial muscles are used to.

The impact of masticatory hypofunctioning on craniofacial muscle development has been discussed in paleoanthropology studies and experimental animal studies.

Masticatory activity is one of the most frequently underestimated functions from both an etiopathogenic and therapeutic perspective in orthodontics. However, the recent development of prospective clinical studies allows for a complementary approach to the correlations between form and function and also allows us to examine the therapeutic importance of high masticatory stress.

\section{KEYWORDS}

Mastication, transverse maxillary growth, masticatory stress

\section{INTRODUCTION}

The development of functional and physiological attritional mastication in children is a necessary condition for the growth and development of the maxilla, which is no longer possible because of the typical consistency of the modern diet.

Orthodontic therapy can alleviate this hypofunction and allow maxillary expansion, but cannot permanently fix the related stabilization issues. The prospective clinical study we present here shows that the targeted introduction of strong masticatory forces can be of great assistance to this therapeutic challenge.

There is a series of anthropometric studies that demonstrate the correlation between the changes in the consistency of food, craniofacial development, and the onset of occlusions. They are based on the comparison of anthropometric data between human skulls found in archeological sites and the skulls of modern day humans. These comparisons have been made between human skulls from the pre-industrial revolution period to

Address for correspondence:

Masrour Makaremi - 2 rue des Deux Conils

24100 Bergerac - France

E-mail: makaremi.orthodontie@gmail.com

Article received: 28-09-2014. Accepted for publication: 29-10-2014.

This is an Open Access article distributed under the terms of the Creative Commons Attribution License (http://creativecommons.org/licenses/by/4.0), which permits unrestricted use, distribution, and reproduction in any medium, provided the original work is properly cited. 
those today $5,21,7,15,17,23-29$. The conclusion of these studies is that the prevalence of malocclusions and craniofacial growth anomalies is much lower in populations with a diet including hard-consistency food than in those with a diet including soft-consistency food.

A complementary approach established by epidemiological studies shows that the frequency of malocclusions has increased in modern populations whose diet has evolved from one with hard-consistency food to that with soft-consistency food ${ }^{8}$.

The problem with anthropological and epidemiological studies is to isolate the influence of the masticatory muscles from other parameters that may affect craniofacial growth (such as an increase in the prevalence of allergies that can lead to oral respiration ${ }^{1}$ ).

The influence of masticatory forces on facial development has also been highlighted by various experiments on animals (Fig. 1). In these experiments, we changed the consistency of food given to young test animals which is then analyzed and compared with growth patterns. Different animals have been examined: $\operatorname{rats}^{2,3,4,14,22,13 \text {; } \text { pigs }^{16} \text {; macaques }}{ }^{6}$; baboons $^{7}$; ferrets ${ }^{10}$; rock hyrax ${ }^{15}$ (the last study is the only experimental one on a retrognathic human-like animal). With these models, we can draw the following general conclusion: an increase in masticatory stresses influence the craniofacial growth. The degree of this influence depends on craniofacial anatomy, and it is difficult to extrapolate these conclusions to human beings because of anatomical differences. However, the animal studies highlight two important elements of retrognathic animals: although the impacts of changes to dietary consistency is more apparent in the lower facial region, there is little effect on the overall skull structure. Above all, the maxillary alveolar transverse dimension (intermolar distance) was consistently altered when the diet is changed to a softer consistency.

Therefore, the relationship between masticatory forces and cranial morphology is the subject of many new studies. Very few studies have tried to introduce stronger masticatory forces as a therapeutic means. However, from the conclusions we have just seen, it could greatly assist us in our objective to develop facial harmony.

Ingervall et al. ${ }^{12}$ emphasized the change in vertical growth direction in hyperdivergent subjects following chewing exercises; Ohira et al. ${ }^{18}$ and He et al. ${ }^{11}$ demonstrate that the introduction of strong masticatory forces significantly increases the physiology of the chewing muscles. In this research area, the following pilot study seeks to determine whether the introduction of strong masticatory forces may not actually help stabilize the transverse alveolar expansion of the arches, which is a serious issue in orthodontics. This idea is worse than the conclusions drawn from the different models of animal experimentation, where the consistency of chewed foods has a systematic impact on the maxillary alveolar transverse dimension (intermolar distance), a diet with hard-consistency food generates more growth in this dimension.

The study is a comparative analysis of Quad Helix alveolar expansion and its development between control subjects and subjects performing intense daily chewing exercises. 


\begin{tabular}{|c|c|c|c|c|c|c|c|}
\hline Animals & Interests & Criticisms & Skull shape & Vertical & Transverse & Sagittal & Alveolar \\
\hline Rats & $\begin{array}{l}\text { - Easy } \\
\text { To manipulate } \\
\text {-known genetic } \\
\text { parameters }\end{array}$ & $\begin{array}{l}\text {-Anatomy } \\
\text { significantly } \\
\text { different to that } \\
\text { of humans }\end{array}$ & $\begin{array}{l}\text { No significant } \\
\text { change between the } \\
\text { two groups }\end{array}$ & $\begin{array}{l}\text { SF: } \\
\text {-Increased Vertical } \\
\text { Dimension }\end{array}$ & $\begin{array}{l}\text { SF: } \\
\text { - Maxillary } \\
\text { transverse } \\
\text { Dimension and } \\
\text { decreased } \\
\text { intermolar }\end{array}$ & $\begin{array}{l}\text { SF: } \\
\text { - Decreased } \\
\text { mandibular sagittal } \\
\text { dimension }\end{array}$ & $\begin{array}{l}\text { HF: - Increased } \\
\text { periodontal } \\
\text { space }\end{array}$ \\
\hline Pigs & $\begin{array}{l}\text { - Animals } \\
\text { + extrapolatable } \\
\text { to humans } \\
\text { - Omnivore } \\
\text { - Orientation } \\
\text { Vertical } \\
\text { mastication }\end{array}$ & $\begin{array}{l}\text { - High Cost } \\
\text { - difficulty } \\
\text { handling } \\
\text { - low number of } \\
\text { specimens }\end{array}$ & $\begin{array}{l}\text { HF: } \\
\text {-Skull } \\
\text { horizontal }\end{array}$ & Not mentioned & $\begin{array}{l}\text { HF: } \\
\text { - largest } \\
\text { interzygomatic dist } \\
\text { ance }\end{array}$ & Not mentioned & $\begin{array}{l}\text { SF: } \\
\text { - Incisive } \\
\text { in range }\end{array}$ \\
\hline $\begin{array}{r}\text { Nonhum } \\
\text { an primate }\end{array}$ & $\begin{array}{l}\text {-Similar to } \\
\text { humans }\end{array}$ & $\begin{array}{l}\text { Difficult } \\
\text { experimental } \\
\text { control parameters }\end{array}$ & $\begin{array}{l}\text { SF: } \\
\text { - Greater cranial } \\
\text { morphological } \\
\text { variability }\end{array}$ & $\begin{array}{l}\text { SF: } \\
\text {-Palatal height } \\
\text { sharply decreased }\end{array}$ & $\begin{array}{l}\text { SF: } \\
\text { - Decreased } \\
\text { maxillary arch } \\
\text { width }\end{array}$ & Not mentioned & $\begin{array}{l}\text { SF: } \\
\text {-Mandibular } \\
\text { obstruction }\end{array}$ \\
\hline $\begin{array}{c}\text { The } \\
\text { importance of } \\
\text { elevated } \\
\text { masticatory } \\
\text { forces on the } \\
\text { stability of } \\
\text { maxillary } \\
\text { expansion } \\
\text { M. Ferrets }\end{array}$ & $\begin{array}{l}- \\
\text { Specific } \\
\text { anatomy - } \\
\text { experimental } \\
\text { parameters } \\
\text { control }\end{array}$ & $\begin{array}{l}\text { - } 1 \text { Study } \\
\text {-Anatomy is very } \\
\text { different } \\
\text { to humans }\end{array}$ & No significant change & $\begin{array}{l}\text { SF: } \\
- \\
\text { Steep and } \\
\text { hard palate }\end{array}$ & $\begin{array}{l}\text { SF: } \\
\text { - Bizygomatic } \\
\text { distance - } \\
\text { decreased } \\
\text { maxillary } \\
\text { intermolar } \\
\text { distance }\end{array}$ & $\begin{array}{l}\text { No } \\
\text { significant change }\end{array}$ & $\begin{array}{l}\text { SF: } \\
\text { - Reduction in } \\
\text { maxillary } \\
\text { intermolar distance }\end{array}$ \\
\hline Rock Hyrax & - Animal & -1 Study & $\begin{array}{l}\text { SF: } \\
\text { - Significant but } \\
\text { localized movement } \\
\text { on the lower side }\end{array}$ & $\begin{array}{l}\text { No } \\
\text { change }\end{array}$ & $\begin{array}{l}\text { SF: } \\
\text { - Decreased } \\
\text { maxillary } \\
\text { intermolar } \\
\text { Distance }\end{array}$ & Not mentioned & $\begin{array}{l}\text { HF: } \\
\text { - Significant } \\
\text { increase in alveolar } \\
\text { ridges }\end{array}$ \\
\hline
\end{tabular}

Figure 1

Bibliographical synthesis of animal experiments. SF: Soft Foods; HF: Hard foods.

\section{MATERIALS AND METHODS}

\section{Material}

\section{Population}

As part of this pilot clinical study, 20 prospective subjects were selected (Fig. 2). The subjects were on average 9 years old and did not show any major skeletal deformities, but did show a mild maxillary linguoversion that required maxillary expansion when the first molars had erupted. These patients were divided into two homogeneous groups (the two groups had similar average ages and typology). 


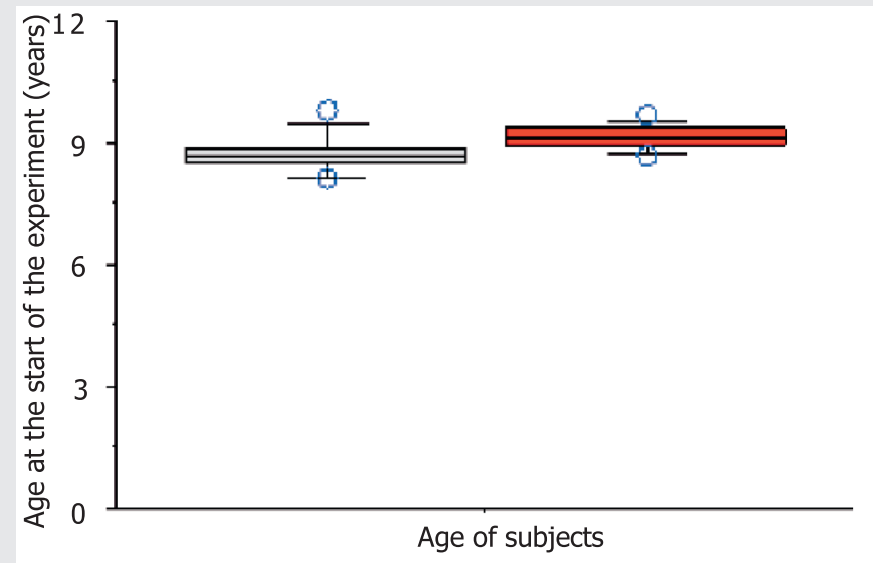

Control

Experiment (GUM)

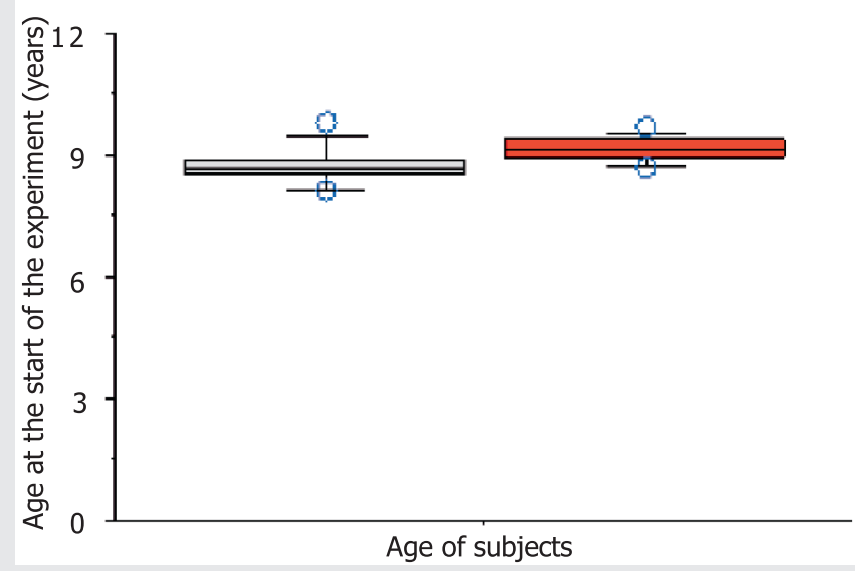

0 Control

Experiment (GUM)

\begin{tabular}{|lr}
\hline Descriptive Statistics \\
Mean & 8.74 \\
Std. Dev & 0.50 \\
Std. Error & 0.16 \\
Count & 10 \\
Minimum & 8.10 \\
Maximum & 9.80
\end{tabular}

Descriptive Statistics: EXPERIMENTAL GROUP (GUM)

Mean $\quad 9.16$

Std.Dev $\quad 0.31$

Std. Error $\quad 0.09$

Count $\quad 11$

Minimum $\quad 8.70$

Maximum $\quad 9.70$

Figure 2

Distribution of subjects by population and age. 


\section{Chewing gum}

The aim was to use chewing gum that generates sufficient stresses to significantly increase the activity of the masticatory muscles. We opted for chewing gum from the island of Chios (masticha or mastiha) which had already been used in Ingervall's experiments et al ${ }^{12}$. It is a semi-transparent resin gum. The resin is extracted from the mastic tree, a tree of the coniferous family. This tree grows only on the island of Chios, Greece. This gum has two advantages: on the one hand, it has been proven to be very effective; on the other hand, there are no ethical issues with its use, as it is a natural product and has been approved for use by the European Union to be sold over-the-counter without any contraindications.

In order to gain a better understanding of the real effect of gum on masticatory muscles, we performed electromyographic (EMG) recordings and three-dimensional motion analysis (Fig. 3) on patients performing chewing exercises with $1.5 \mathrm{~g}$ of the Chios chewing gum and $1.5 \mathrm{~g}$ of classic chewing gum (Hollywood brand). The recordings focused on the masseter muscle during the experimental phase and patients were asked to restrict chewing to the left-hand side of the mouth.

The analysis of the results shows that chewing the harder gum generates a significant increase in muscle activity: on the left side, there is an increase of $93 \%$ in activity of the left masseter muscle whereas there is an increase of $326 \%$ in muscle activity of the right masseter muscle. (This difference is because of the need to stabilize the non-working side given the intensity of occlusal pressure.)

\section{Experimental protocol}

In the first stage of the experiment, all subjects undergo a Quad Helix maxillary arch expansion. In the second stage, the control subjects are kept under surveillance. The other ten subjects are asked to chew the Chios gum. Radiological and anthropometric data are collected throughout the study: lateral and profile skull teleradiography, panoramic dental arches, study models, maximum occlusal strength measurements, and chewing gum film (Fig. 4).

The decision to precede the active chewing phase with a therapeutic phase consisting of maxillary expansion increased the duration of the treatment. However, this phase is likely to introduce bias into the study.

Therefore, the active chewing phase will be spread over 6 months. A longer experiment would have been preferable, however, the radiological data were collected before the first phase, and the growth of the maxillary arch could be analyzed over 1 year, which was sufficient time to study it using teleradiography.

The daily duration of the exercise was $60 \mathrm{~min}$, thereby approaching a situation in which the subject eats hard-consistency foods daily. The exercises did not begin directly after the Quad Helix expansion because, as demonstrated by many different studies $^{19}$, during the active phase of treatment, proprioception and analgesic reflexes greatly decrease the efficiency of the masticatory muscles. A delay of 1 month therefore separated the removal of the Quad Helix from the beginning of the chewing exercises. 

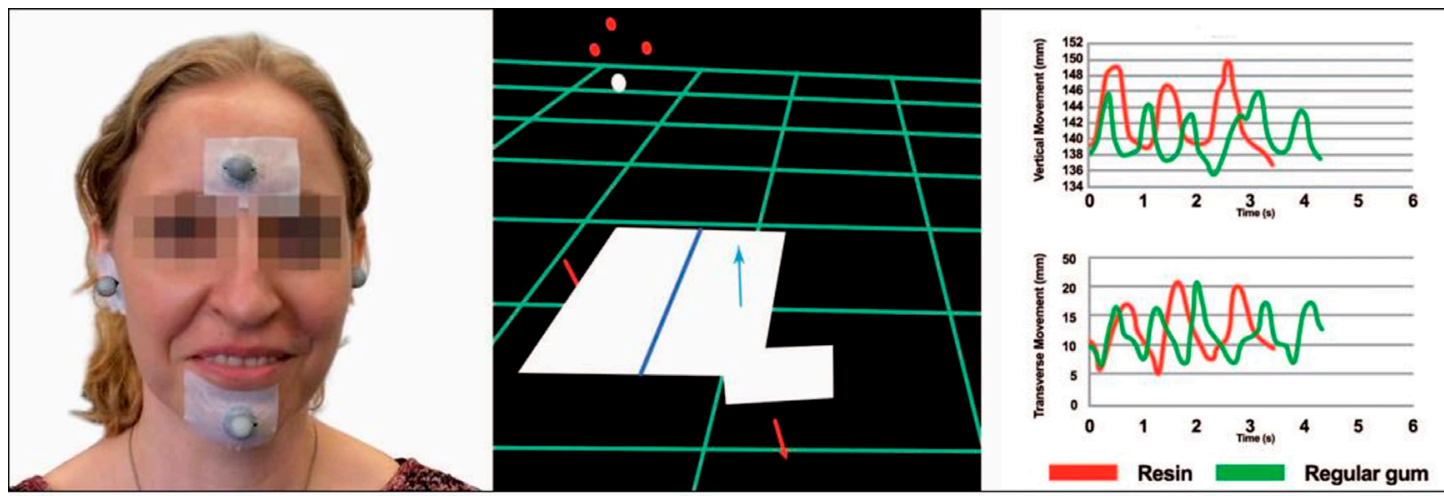

Figure 3

Comparative analysis of mandibular movements when chewing Chios gum (resin) and when chewing Hollywood brand chewing gum (regular gum) using three-dimensional motion sensors.

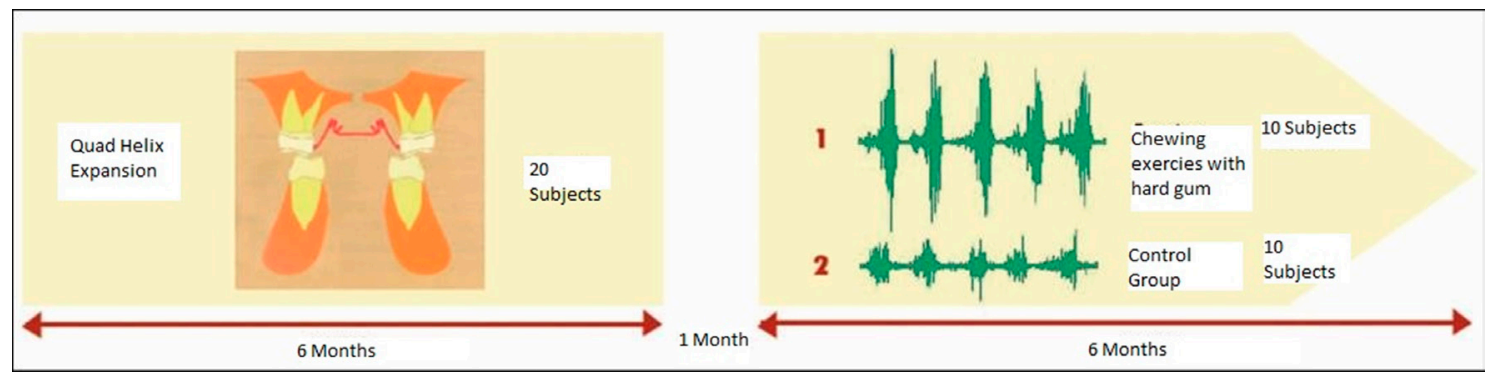

Figure 4

Chronology of the implementation of the experimental protocol.

\section{METHODS}

\section{Data collection}

The study is based on the analysis of the intermolar distance between the first maxillary molars and their orientation in relation to the median sagittal plane.

The intermolar distance was measured using a caliper, between the top of the mesiovestibular cusp of tooth 16 to tooth 26 (paracone), on castings made prior to the Quad Helix implementation. Then this measurement was repeated on other casts after chewing exercises. The detection of the cusp is quite simple and the measurement seems reliable, which means the measurement can easily be reproduced.

The orientation of the first maxillary molars was measured using facial teleradiography, performed before the Quad Helix implementation and after the chewing exercises (teleradiography was performed 1 year apart). The angle is measured between the median sagittal plane and the tangent 
of the vestibular plane at 16 and 26, taking the most convex point. Despite difficulties when reading the facial teleradiography, this measure, based on anatomical elements which can be easily located, could be reproduced (the same radiographic analysis performed by two observers giving the same measurements).

\section{Statistical methodology}

The statistical analysis of the data was then carried out using the Kruskal-Wallis (KW) test. We chose this test, which had been developed by Wilcoxon in 1945 and which is well suited to small samples if it is used in tandem with Siegel's tables. This test can be seen as a generalization of the Wilcoxon-Mann-Whitney test to more than two samples. It is a nonparametric test: it makes no assumptions about the form of the underlying distributions. Like many nonparametric tests, it works not on the values of the observations, but on their rank, once these observations are gathered in a single large sample. This is a test especially suitable for samples with fewer than 15 data entries.

The test is used here to determine whether the two sample groups (control group and chewing group) are from the same population, on a scale measured by a numerical value (occlusal forces, cephalometric value, intermolar distance). So, this is an identity test: if the hypothesis is rejected, then there is a significant difference between the two populations.

\section{RESULTS}

The relationship between daily chewing of hard gum and maxillary transverse growth is more apparent when both intermolar distance and molar orientation are considered (Fig. 5).

The effects on the maxillary transverse dimension are highlighted by the contraction of the maxillary arch, which defines the population of the study (indication of Quad Helix).

The intermolar distance increased proportionately in the population who chewed gum 6 months after the expansion of the maxillary arch, combined with molars that are at a lower level than the median sagittal plane in the control sample. Therefore, in the subjects who chewed hard gum, the masticatory forces improved the stability of the molar expansion and straightened the molar axes. J.L. Raymond $^{20}$ emphasized the importance of controlling molar torque to therapeutic expansion. Controlling molar torque can be best achieved naturally by chewing hard gum; The effects here are measured in a quantitative way so as to be clinically perceptible. In fact, both observers reported that the meshing of the lateral sectors were much more efficient in the subjects who had chewed hard gum than subjects in the control group.

Even if the sample size is small, the net discrimination in the recovery of the molar axes tends to show that this phenomenon can be attributed to chewing hard gum. 
Therefore, there is a significant difference in the transverse dimension on the alveolar plane. It is the direct consequence of the masticatory forces on the face, without any prior modification to the masticatory muscles. The adjustment to the molar axes and a better meshing of the lateral areas (Fig. 6) are clinically beneficial.

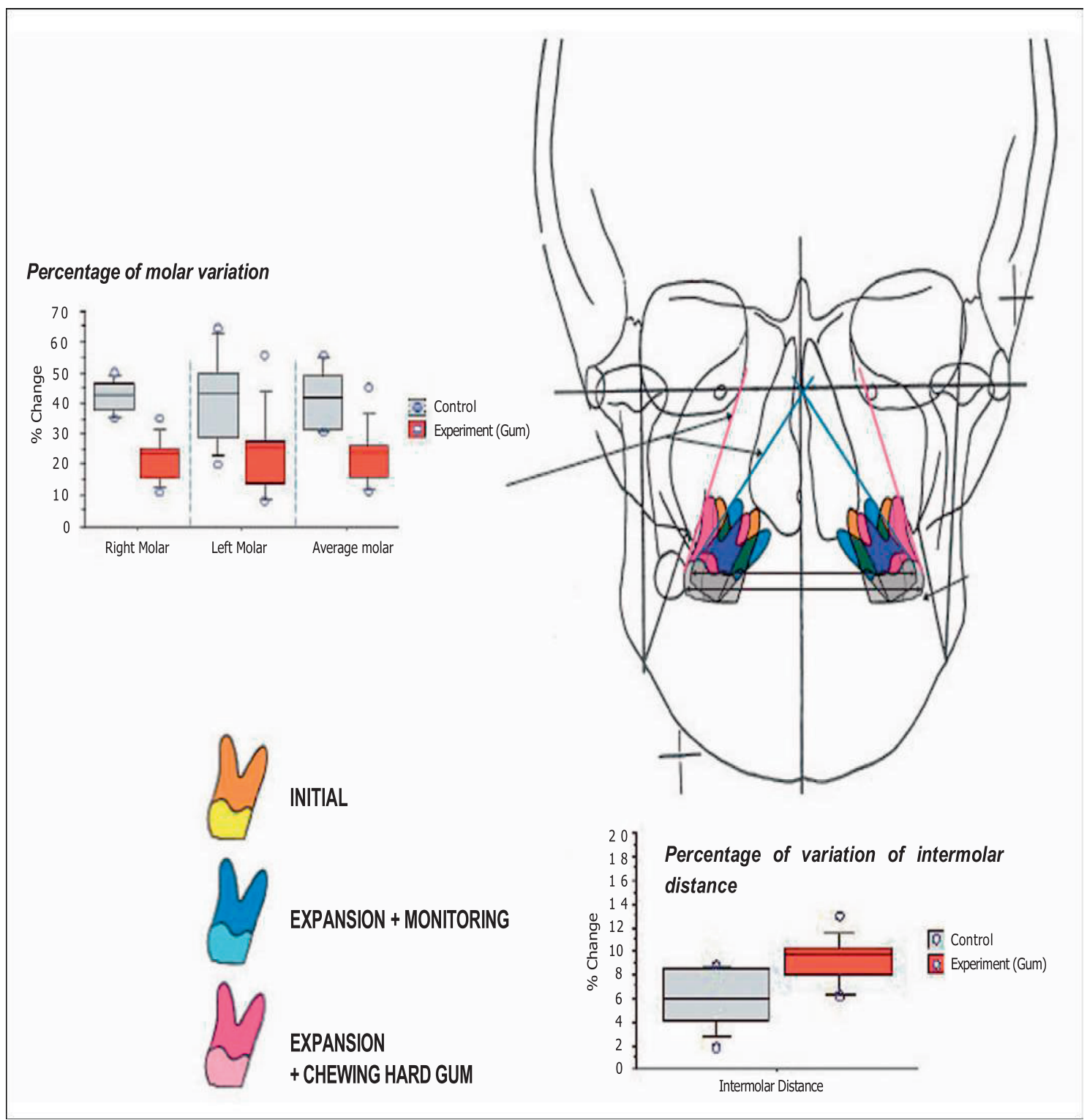

Figure 5

Summary of results. 

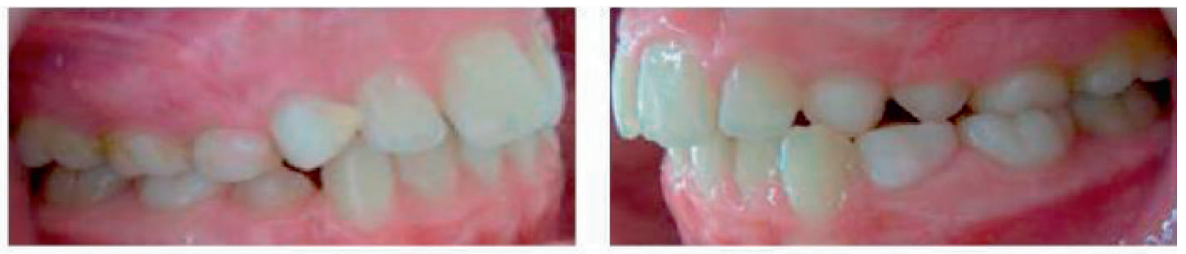

Start of treatment
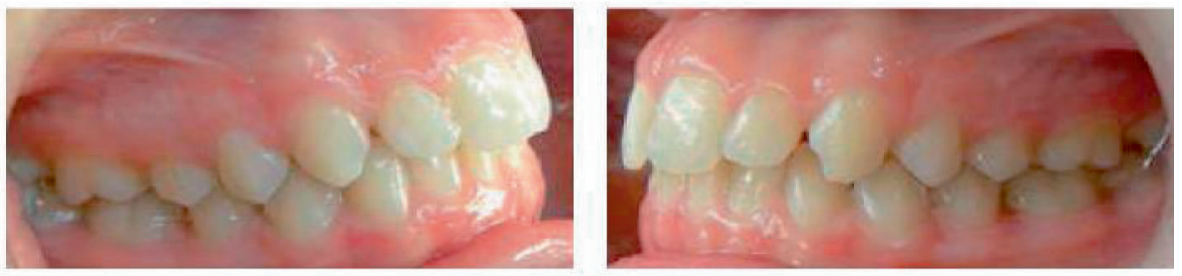

6 months after chewing the gum ( +1.5 years)
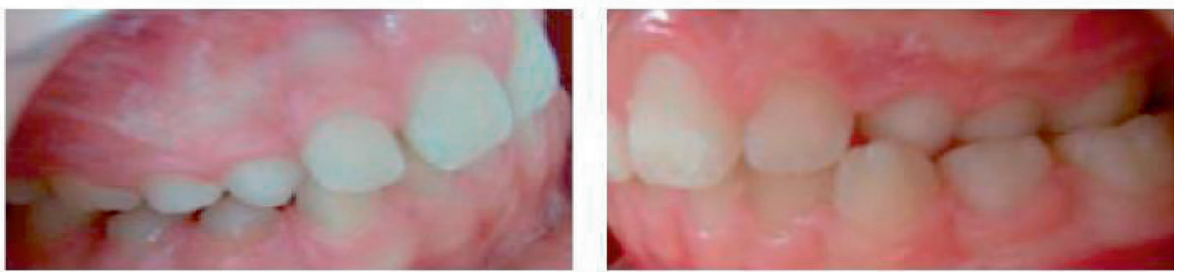

Start of treatment
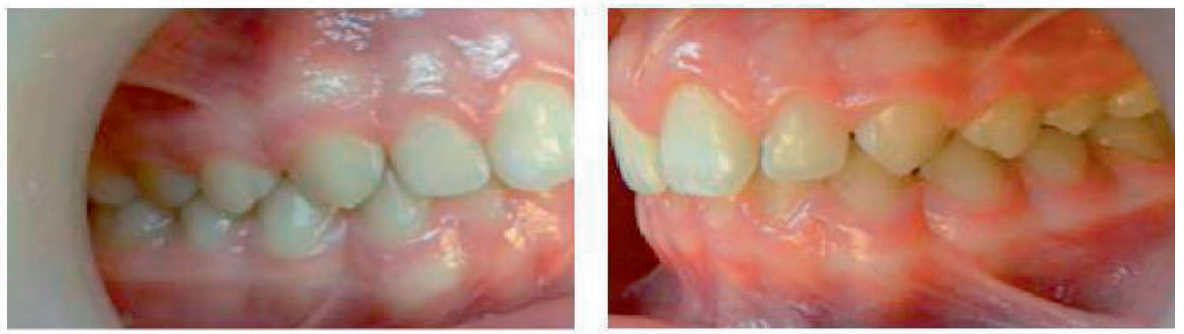

6 months after chewing the gum ( +1.5 years)

Figure 6

\section{DISCUSSION}

We started this study with a Lamarckian approach to the relationship between the masticatory muscles in young patients and the changes in our diet after the industrial revolution.
The relationship between the development and the transverse stability of the dental arches and the consistency of our diet has moved from animal experimentation toward human clinical 
studies. However, this is only a small step which, in many respects, would need to be supported.

Throughout this pilot study, we observed that it would be better to perform a thorough clinical study with a larger sample of young patients who can be monitored over a longer period of time, without any other treatment other than chewing gum.

The results would then be able to assess the efficacy of strong masticatory forces as a therapeutic means to achieve a more balanced craniofacial development. The path to clinical applications is long and needs us to answer many questions along the way in order to develop a more prophylactic orthodontic treatment to care for young patients.

Beyond the quantitative data discussed in this study, there remains a clinical impression felt by the two practitioners who followed the subjects. We felt that the meshing of the dental arches was much better in subjects who had chewed the gum compared to the control sample. This clinical impression merits further research and leads us to believe that the chewing exercises would allow the subject to balance occlusal functions on their own.

\section{CONCLUSION}

The postmodernist philosopher Mr. Foucault stresses: It is not a question of why (cause) but a question of the effects, we must pay close attention to the processes and functions. On the basis of this reasoning, we can look at a contrario the introduction of strong masticatory forces, for which our masticator is naturally designed, as a therapeutic lever.

Conflict of interest: The authors declare that the have no conflict of interest.

\section{BIBLIOGRAPHY}

1. Aberg N, Hesselmar B, Aberg B, Eriksson B. Increase of asthma, allergic rhinitis and eczema in Swedish schoolchildren between 1979 and 1991. Clin Exp Allergy 1995;25:815-819.

2. Barber CG, Green LJ, Cox GJ. Effects of the physical consistency of the diet on the condyle growth of the rat mandible. J Dent Res 1963;42:848-851.

3. Beecher RM, Corruccini RS, Freeman M. Craniofacial correlates of dietary consistency in a nonhuman primate. J Craniofac Gen Dev Biol 1983;3:193-203.

4. Bouvier M, Hylander W. Effect of bone strain on cortical bone structure in macaques (M. mulatta). J Morphol 1981;167:1-12.

5. Carlson DS, Van Gerven DP. Masticatory function and Post Pleistocene evolution in Nubia. Am J Phys Anthrop 1977;46:495-506.

6. Ciochon RL, Nisbett RA, Corruccini RS. Dietary consistency and craniofacial development related to masticatory function in minipigs. J Craniofac Genet Dev Biol 1997;17:96-102. 
7. Coruccini RS, Beecher R. Occlusal variation related to soft diet in a nonhuman primate. Science 1982;218:7476.

8. Coruccini RS, Beecher R. Occlusofacial morphological integration lowered in baboons raised on soft diet. J Craniofac Genet Dev Biol 1984;4,135-142.

9. Harper CA. Comparison of medieval and modern dentitions. Eur J Orthod 1994;16: 163-173.

10. He T, Kiliaridis S. Effect of masticatory muscle function on craniofacial morphology in growing ferrets. Eur J Oral Sci 2003;111:510-517.

11. He T, Stavropoulos D, Hagberg C, Hakeberg M, Mohlin B. Effects of masticatory muscle training on maximum bite force and muscular endurance. Acta Odontol Scand 2013 MayJul;71(3-4):863-9.

12. Ingervall $B$, Bistanis $E$. A pilot study of the effect of masticatory muscle training on facial growth in long-face children, Eur J Orthod 1987;9:15-23.

13. Katsaros $C$, Berg R, Kiliaridis $S$. Influence of masticatory muscle function on transverse skull dimension in the growing rat. J Orofac Orthop 2002;63:5-13.

14. Kiliaridis S, Shyu BC. Isometric muscle tension generated by masseter stimulation after prolonged alteration of the consistency of the diet fed to growing rats. Arch Oral Biol 1988;33:467-472.

15. Lieberman DE, Krovitz GE, Yates FW, Maureen D, St Claire M. Effects of food processing on masticatory strain and craniofacial growth in retrognathic face. J Human Evolution 2004;46:655-677.

16. Lindsten R, Magnusson T, Ogaard B, Larsson E. Effect of food consistency on temporomandibular joint morphology: an experimental study in pigs. J Orofac Pain 2004;18:56-61.

17. Mohlin B, Sagne S, Thilander B. The frequency of malocclusion and the craniofacial morphology in a medieval population in southern Sweden. O.S.S.A. 1978;5:57-84.

18. Ohira $A 1$, Ono Y, Yano $N$. The effect of chewing exercise in preschool children on maximum bite force and masticatory performance. Takagi Y. Int J Paediatr Dent 2012 Mar;22(2): 146-53.

19. Petrén S1, Bjerklin K, Bondemark L. Stability of unilateral posterior crossbite correction in the mixed dentition: a randomized clinical trial with a 3-year follow-up. Am J Orthod Dentofacial Orthop 2011;139(1):e73-81.

20. Raymond J-L. Mastication et corrections occlusales transversales. Rev Orthop Dento Faciale 2001;35:339346.

21. Sardi ML, Novellino PS. Craniofacial morphology in the Argentine Center West: Consequence of the transition to food production. Am J Phys Anthropol 2006;130:333-343.

22. Ulgen M, Baran S, Kaya H, Karadede I. The influence of the masticatory hypofunction on the craniofa-cial growth and development in rats. Am J Orthod Dentofacial Orthop 1997;111:189-198.

23. Varrela J. Effects of attritive diet on craniofacial morphology: a cephalometric analysis of a finish skull sample. Eur J Orthod 1990;12:219-223. 